\title{
LA POBREZA ENERGÉTICA DESDE UNA PERSPECTIVA DE GÉNERO EN HOGARES URBANOS DE ARGENTINA ${ }^{1}$
}

\author{
María Eugenia Castelao Caruana* \\ Florencia Magdalena Méndez ${ }^{* *}$
}

Resumen. El artículo explora el alcance e incidencia de la pobreza energética de los hogares urbanos en Argentina y su relación con la división de tareas en el interior del hogar entre los géneros. Para ello indaga en los enfoques conceptuales y metodológicos utilizados a nivel internacional para abarcar este fenómeno socio-económico, y analiza la calidad de la información disponible en el país para medir la pobreza energética y estimar su incidencia en los hogares y, en particular, en el trabajo no remunerado de las mujeres. Este análisis pone de relevancia dos dimensiones claves de la pobreza energética en los hogares urbanos: el acceso a servicios energéticos modernos y confiables, y la disponibilidad de los bienes económico que median en el consumo energético para responder a satisfactores como la alimentación, el aseo personal, el confort térmico, la seguridad, el entretenimiento y la comunicación. En Argentina, la pobreza energética afecta con mayor intensidad a aquellos hogares urbanos que, al mismo tiempo, utilizan más horas de trabajo doméstico y de cuidado no remunerado, tareas realizadas principalmente por mujeres.

Palabras Clave: Pobreza energética; Género; Uso del tiempo

\footnotetext{
* Centro de Estudios Urbanos y Regionales, Consejo Nacional de Investigaciones Científicas y Técnicas (CONICET), Argentina

Contacto: eugeniacastelao@conicet.gov.ar

** Centro de Estudios Urbanos y Regionales, Consejo Nacional de Investigaciones Científicas y Técnicas (CONICET), Argentina
}

Contacto: mendez.magdalena@conicet.gov.ar

1 Una versión anterior a este artículo fue presentada en las XII Jornadas Nacionales de Investigadores en Economías Regionales, Quilmes, Argentina. 


\title{
ENERGY POVERTY WITH A GENDER PERSPECTIVE IN URBAN HOUSEHOLDS IN ARGENTINA
}

\begin{abstract}
This article shows the energy poverty in urban households and its impact on gender inequality in the use of time in Argentina. It explores the scope of the different conceptual and methodological approaches to this socio-economic problem as well as the quality of the information available in the country to measure energy poverty and its effect on women. The study analyses, particularly, the incidence of two key dimensions of energy poverty in urban households in Argentina: access to modern and reliable energy services, and availability of the economic goods that intervene in the consumption of energy, such as feeding, personal hygiene, thermal comfort, safety, and entertainment and communication. Country level analysis reveals that this issue affects more intensely the urban households that use more hours of unpaid domestic and care work, which are tasks mainly performed by women.
\end{abstract}

Keywords: Energy Poverty; Gender; Use of time

Original recibido el 03/10/2018

Aceptado para su publicación el 18/06/2019 


\section{Introducción}

El acceso universal a servicios de energía limpios, confiables y asequibles es una meta necesaria para impulsar el desarrollo y la sustentabilidad ambiental (United Nations, 2018). Esta meta forma parte de los Objetivos de Desarrollo Sostenible (ODS), pero su incidencia en muchos de los objetivos propuestos por esta agenda le confiere un alto nivel de reconocimiento político y un papel central en la agenda de desarrollo. En América Latina y el Caribe (ALyC) alrededor de 21,8 millones de personas carecen de acceso a electricidad (Rojas y Siles, 2017), pero un número aún no estimado de personas se encuentran en condiciones de pobreza energética.

El consumo de energía moderna ${ }^{2}$ en el ámbito doméstico permite a los hogares cubrir necesidades básicas, mejorar la calidad ambiental y participar en prácticas de consumo, habituales y esperadas, con significado social y cultural (Liddell y Morris, 2010; Sovacool, Sidortsov y Jones, 2014). Sin embargo, la pobreza energética, entendida como la imposibilidad de los hogares de consumir un nivel adecuado de energía para satisfacer sus necesidades fundamentales a través de tecnologías modernas, es un problema presente en la mayoría de los países del mundo. En los países más pobres, este problema se manifiesta esencialmente en la ausencia de fuentes primarias de energía y/o de tecnología moderna para explotarlas. En los países con índices de desarrollo más altos, la pobreza energética se encuentra asociada al costo de la energía, el ingreso de los hogares, la eficiencia de las viviendas y las convenciones culturales de cada sociedad.

Las estrategias que despliegan los hogares para hacer frente a la pobreza energética poseen una dimensión de género ${ }^{3}$ ineludible al analizar los aspectos de este problema y la formulación de políticas que lo aborden. Históricamente, en la división sexual del trabajo, las mujeres han sido las principales responsables de la gestión cotidiana del hogar y del cuidado de la familia y, por ende, de las actividades y los recursos que permiten sobrellevar la pobreza energética. La recolección y/o compra de leña o combustible líquido para cocinar y calefaccionar la vivienda, el lavado manual de ropa, la recolección de agua, entre otras actividades domésticas (ECOWAS, 2015), son tareas derivadas de la condición de pobreza energética en la que se encuentran los hogares, que impactan en el bienestar físico de las personas y en su capacidad de desarrollar una vida plena. De esta forma, la pobreza energética puede contribuir a la feminización de la pobreza al incrementar el tiempo que las mujeres dedican a actividades domésticas y de cuidados no remuneradas (Simcock y Mullen, 2016). A su vez, la sobrecarga de los tiempos de trabajo puede deteriorar la salud de las mujeres y/u obligarlas a resignar otras actividades tanto reproductivas como productivas remuneradas.

\footnotetext{
2 Siguiendo los ODS, el término energía moderna refiere a la energía generada y/o provista mediante tecnología limpia, de bajo impacto para la salud de las personas y el ambiente, incluyendo el combustible y los artefactos utilizados para cocinar (United Nations, 2018).

3 Se piensa el género en base a la definición de Scott (1993) que se compone de "una conexión integral entre dos proposiciones: el género es un elemento constitutivo de las relaciones sociales basadas en las diferencias que distinguen los sexos y el género es una forma primaria de relaciones significantes de poder" (págs. 34-35).
} 
El presente estudio se propone avanzar sobre el análisis de la pobreza energética que enfrentan los hogares urbanos en Argentina y su incidencia en el uso del tiempo en el hogar. Para ello se indaga en los distintos enfoques conceptuales y metodológicos utilizados para comprender este fenómeno y en el alcance de la información disponible en Argentina para medir y estimar su incidencia en la distribución del tiempo en el hogar según género.

El trabajo se organiza en cuatro apartados. A continuación, se presenta un breve apartado metodológico, el cual explicita las bases de datos que se utilizarán en este artículo con una caracterización de las principales variables que indaga cada una. Luego se presentan los antecedentes teóricos en torno al concepto de pobreza energética, a la vez que se esquematiza su vinculación con el género. Por otro lado, se analizan las bases de datos a nivel de hogar disponibles en el país y su capacidad de aportar información para estudiar este problema. Se presentan, además, datos empíricos que permiten indagar sobre una dimensión de la pobreza energética -el acceso a bienes económicos que median en el uso de la energía- y su incidencia en el uso del tiempo por género. Para finalizar, se reflexiona sobre los principales hallazgos del estudio y se aporta a la discusión en torno a la noción de pobreza energética y las desigualdades de género que esta genera.

\section{Metodología}

Para analizar la calidad de la información disponible en el país y, posteriormente, construir un indicador de pobreza energética con perspectiva de género, se tomaron tres bases de datos. La primera es el Censo Nacional de Población, Hogares y Viviendas (Censo) del año 2010 (INDEC [Instituto Nacional de Estadística y Censos], 2015) que, junto al cuestionario ampliado, releva los principales aspectos demográficos, sociales y habitacionales de la población. Permite acceder a información sobre la calidad constructiva de las viviendas, el acceso a servicios básicos, y a equipamiento doméstico como heladera, computadora y teléfono celular. Otra base es la Encuesta Anual de Hogares Urbanos (EAHU), que cubre 31 aglomerados urbanos del país con más de 2.000 habitantes e investiga principalmente la dinámica del mercado laboral. En el año 2013, esta encuesta incorporó un módulo sobre Trabajo No Remunerado y Uso del Tiempo (INDEC, 2014), con el objetivo de captar información respecto de la participación y el tiempo destinado por las personas de 18 años y más a tareas domésticas, el cuidado de miembros del hogar y el trabajo voluntario.

Por último, se analiza la Encuesta Nacional sobre Estructura Social (ENES) realizada entre el segundo semestre de 2014 y el primer semestre de 2015 por el Programa de Investigación sobre la Sociedad Argentina Contemporánea (PISAC). La ENES es de alcance urbano nacional y se aplicó a una muestra de 12.000 hogares. El abordaje de la caracterización social del hogar se deriva en términos operativos de los atributos de uno o algunos de sus miembros. Esta encuesta tiene como principal objetivo conocer los aspectos estructurales de la heterogeneidad social, tales como composición del hogar; vivienda y hábitat; vulneración de derechos alimentación y nutrición; acceso a bienes, servicios y ayudas de otros; bancarización y acceso al crédito; educación; salud; ingresos. Adicionalmente al indagar el trabajo, 
esta encuesta adopta una perspectiva de género (PISAC, 2015), analizando la participación de las personas en el trabajo doméstico y de cuidados de miembros del hogar dependientes (Maceira, 2015).

La elección de las encuestas respondió a la decisión de indagar la incidencia de la pobreza energética en las desigualdades de género a partir del tiempo destinado por los miembros del hogar al trabajo doméstico y de cuidados. El Censo, por otra parte, ofrece resultados de alcance nacional, válidos a nivel de departamento y, por lo tanto, más confiables que los datos obtenidos a nivel de aglomerados urbanos. De esta forma, los datos del Censo son una referencia válida y confiable para el análisis de otras bases de datos teniendo en cuenta su diferencia temporal.

\section{De la pobreza de combustible a la pobreza energética}

El término pobreza de combustible (fuel poverty) comienza a ser utilizado en Europa a comienzos del siglo XX para hacer referencia a la situación que atraviesan los hogares que no poseen ingresos suficientes para adquirir el combustible necesario para iluminar, cocinar y mantener el confort térmico en su vivienda (Richardson, 1978; Lewis, 1982). Años después, Boardman (1991) propone que un hogar se encuentra en condiciones de pobreza de combustible cuando gasta más del $10 \%$ de su ingreso en diversas fuentes de energía. Esta propuesta pondera la eficiencia energética de las viviendas y de esta forma el rol de las tecnologías aplicadas a la generación de calor y la aislación térmica, pero resulta sumamente sensible a cambios en el precio de los combustibles (Moore, 2012). Desde los ochentas se han elaborado diversas definiciones del término pobreza de combustible en Europa que, según Papada y Kaliampakos (2016), pueden resumirse en la incapacidad de un hogar para cubrir adecuadamente sus necesidades energéticas debido a su bajo nivel de ingreso, el costo de la energía y la eficiencia energética de la vivienda.

La extensión de la noción de pobreza de combustible, desde el acceso a confort térmico en las viviendas en los países desarrollados hacia el acceso a energía a través de tecnologías modernas y confiables en los países en desarrollo, encuentra su correlato en el surgimiento del término pobreza energética. En los países europeos estos términos son utilizados indistintamente (Thomson, Snell y Liddell, 2016), pero en ALyC el término pobreza energética es preponderante. Sin embargo, ambos comparten una condición común: la imposibilidad de los hogares de obtener los niveles materiales y sociales de energía necesarios para su subsistencia (Belaïd, 2018).

A lo largo de los años, la investigación de la pobreza energética se ha desarrollado en torno a dos enfoques teóricos y metodológicos centrales para el estudio de la pobreza: el enfoque de subsistencia y el enfoque consensual (García-Ochoa y Graizbord, 2016). Los trabajos que adoptan el enfoque de subsistencia centran su análisis en la interacción entre el nivel de ingreso de los hogares, la aislación térmica y los precios de la energía, incluyendo en el análisis factores como el equipamiento de las viviendas, las características sociodemográficas del hogar y sus hábitos de consumo, entre otros (Thomson, Snell, y Bouzarovski, 2017).

El estudio sobre privación relativa de Townsend cuestiona el enfoque de subsistencia en el análisis de la pobreza por la centralidad que otorga a las necesidades físicas 
por sobre las sociales. Sugiere que las carencias que enfrentan las personas deben analizarse considerando la estructura social e institucional en cada lugar y tiempo determinado. Esta estructura otorga a las personas roles sociales y económicos que condicionan sus formas de consumo material. A su vez, los bienes materiales que satisfacen las necesidades se encuentran socialmente determinados y pueden modificarse con el tiempo (Townsend, 2006). Con base en este enfoque, Healy y Clinch (2002) desarrollaron una metodología para medir la pobreza energética que incluye aspectos objetivos, como el equipamiento de la vivienda, y subjetivos como la percepción de las personas sobre su situación de pobreza energética. De esta forma, la pobreza energética es analizada como una condición relativa respecto a las necesidades consideradas básicas e indispensables en cada sociedad (GarcíaOchoa, 2014).

En ALyC la pobreza energética es una noción emergente para analizar un problema histórico, que excede la incapacidad de los hogares de lograr niveles adecuados de confort térmico. En la región, el uso del término abarca tanto la falta de acceso a energía mediante sistemas modernos y confiables como la imposibilidad de los hogares de utilizar la energía para satisfacer sus necesidades físicas y sociales (transporte, iluminación, cocción, comunicación, etc.). En este sentido, la pobreza energética permite dar cuenta del papel central que posee la energía para alcanzar los ODS (Modi, Mc Dade, Lallement y Saghir, 2005; Mininni, 2015).

A partir del método de Necesidades Básicas Insatisfechas presentado por la Comisión Económica para América Latina y el Caribe (CEPAL) para medir la pobreza en la década del ochenta, los aportes de Sen (1981) al debate sobre privaciones y capacidades, y la distinción de Max Neef, Elizalde y Hopenhayn (1986) entre necesidades, satisfactores y bienes económicos, García Ochoa (2014) propone un enfoque centrado en las Necesidades Absolutas de la Energía para analizar la pobreza energética en ALyC. Este enfoque distingue entre necesidades, satisfactores y bienes económicos. Las necesidades son absolutas, finitas, clasificables e iguales en todas las culturas y períodos históricos e incluyen dimensiones como la subsistencia, la protección, la participación, el ocio, la identidad, la libertad, etc. Los satisfactores contribuyen a la realización de estas necesidades y a la vez las expresan, y cambian con el contexto histórico, social y cultural (Boltvinik Kalinka, 2005). En materia energética, algunos de los satisfactores considerados por García-Ochoa (2014) son alimentación, trabajo, descanso, cuidado (atención de la persona), tiempo libre, salud física y mental, etc. Los bienes económicos, por último, median en la realización de estos satisfactores y también dependen del entorno social y el momento histórico. El análisis del proceso de creación, mediación y condicionamiento entre necesidades, satisfactores y bienes económicos permite entender cómo se perciben las necesidades y cómo la interacción entre satisfactores y bienes económicos modelan las estrategias de supervivencia de las personas y sus hogares (Boltvinik Kalinka, 2005).

El enfoque de las Necesidades Absolutas de la Energía propone medir la pobreza energética a partir del acceso de los hogares a los bienes económicos que consumen energía y permiten hacer un uso práctico de este servicio para satisfacer las 
necesidades absolutas. Entre los bienes económicos que identifica García-Ochoa (2014) se encuentran heladera, computadora, acceso a internet, calentador de agua de gas o eléctrico, ventilador, aire acondicionado o calefactor (según el clima de la región), lámpara, televisión y horno a gas o eléctrico.

En resumen, al analizar el alcance y los factores que condicionan la pobreza energética en la región, así como su impacto económico, social y ambiental, es necesario contemplar las múltiples dimensiones que condicionan la satisfacción de las necesidades absolutas de los hogares a través del consumo de energía. En este sentido, la pobreza energética en ALyC es resultado, por un lado, de la falta de acceso a servicios energéticos modernos y confiables y, por otro lado, de la interacción entre las tarifas de energía, la eficiencia energética de las viviendas -su infraestructura y equipamiento- y el ingreso de los hogares. Por último, entre la situación de acceso y el consumo efectivo de energía se encuentran los bienes económicos que permiten y/o facilitan la satisfacción de necesidades. Todos estos factores, a su vez, están condicionados por el entorno, esto es por relaciones sociales, productivas y de género y condiciones ambientales (urbano-rural, clima, geografía, etc.) que moldean los hábitos de consumo.

Debido a su carácter multicausal, la pobreza energética es una condición que puede afectar tanto a los hogares que gastan un alto porcentaje de sus ingresos en energía como a quienes gastan montos bajos en términos absolutos. Esta situación, a su vez, puede reflejar tanto una decisión voluntaria de restringir el consumo de energía como la falta de acceso a los bienes económicos básicos que median en su consumo.

\section{Pobreza energética y género}

El empoderamiento de las mujeres y la igualdad de género fueron considerados metas claves para alcanzar los ODS en el año 2000. El "acceso a una energía asequible, fiable, sostenible y moderna para todos" (United Nations, 2018) fue incorporado como tema prioritario en esta agenda quince años después, destacando su importancia en materia de alimentación, calefacción, iluminación, comunicación y producción (Minini, 2015). Esta meta propone cambios en los niveles y patrones de consumo de energía a nivel global e impulsa la transición energética. Sin embargo, pocos proyectos reconocen que los beneficios y costos de estos cambios -como la generación distribuida de energía renovable, por ejemplo- pueden incidir desigualmente al interior del hogar (Khamati-Njenga y Clancy, 2003; Simcock y Mullen, 2016). En otras palabras, las medidas implementadas y los resultados obtenidos en materia de energía y género en el marco de los ODS aún son analizados de manera disociada, sin considerar la transversalización de género ${ }^{4}$.

La división de tareas en el interior de los hogares asigna un rol fundamental a las mujeres como responsables de la gestión del hogar y el cuidado de la familia. La feminización en el acceso a la energía depende de los trabajos que se realizan en los hogares y la manera en que se resuelven: cuidado de niños/as, limpieza

4 En 1997 el Consejo Económico y Social de las Naciones Unidas definió el concepto de tranversalización de la perspectiva de género como "el proceso de valorar las implicaciones que tiene para los hombres y para las mujeres cualquier acción que se planifique, ya se trate de legislación, políticas o programas, en todas las áreas y en todos los niveles". 
de la vivienda con o sin la participación de empleados/as domésticos/as, trabajo domiciliario, presencia de adultos/as mayores, etc. (González Pijuan, 2017). Estos determinan las actividades que desarrollan las mujeres en torno a la organización del hogar y, consecuentemente, la incidencia dispar de la pobreza energética. Este fenómeno se refleja en la recolección de combustible leñoso para cocinar y/o calefaccionar las viviendas, la cocción con estufas ineficientes, el lavado a mano de ropa, la gestión del alimento sin heladera o frente a cortes de luz prolongados, etc. (Rojas y Siles, 2014).

El análisis de la pobreza energética atendiendo a las desigualdades de género se centra en descomponer la interacción entre los servicios energéticos y las relaciones de género subyacentes al interior de los hogares (Skutsch y Wamukonya, 2001). Este enfoque no solo examina los beneficios económicos de los servicios de energía, sino también la manera en que estos contribuyen a mejorar el bienestar de las personas (Munien y Ahmed, 2012). En este marco, el enfoque de las Necesidades Absolutas de la Energía adquiere relevancia para comprender el uso de los bienes económicos y su impacto sobre los tiempos de trabajo doméstico y de cuidado no remunerado. Por ejemplo, la tenencia de un lavarropa y su incidencia en el uso del tiempo de las mujeres no es igual en una familia extensa que en una familia nuclear sin hijos/as.

Como unidad de análisis, la familia esconde situaciones reales de desigualdad como la distribución de las tareas del hogar y el acceso al mercado de trabajo remunerado, entre otros. En los hogares urbanos de Argentina se observa con claridad una mayor participación de las mujeres en todas las tareas domésticas y de cuidado no remuneradas, pero en especial en actividades como limpieza de la casa (81\%), preparación de las comidas (72\%) y realización de las compras $(74 \%)$ (Tabla 1).

Tabla 1. Porcentaje de personas que manifestaron haber realizado trabajo doméstico y de cuidado no remunerado durante la semana anterior a la encuesta, según género.

\begin{tabular}{|l|c|c|c|}
\hline $\begin{array}{l}\text { Tipos de trabajo doméstico y de cuidado } \\
\text { no remunerado }\end{array}$ & Varón & Mujer & Otro* \\
\hline Limpiar y ordenar la casa & 46 & 81 & 100 \\
\hline Planchar & 15 & 55 & 45 \\
\hline Hacer la comida & 39 & 72 & 86 \\
\hline Hacer las compras & 61 & 74 & 68 \\
\hline Hacer trámites o pagos & 50 & 58 & 33 \\
\hline
\end{tabular}

Nota: $\left(^{*}\right)$ Esta encuesta adopta una definición abierta del sistema de género, incluyendo en la categoría "Otro", múltiples identidades relevadas mediante auto-designación (Maceira, 2015). Esta categoría representa el $0,01 \%$ de la población estimada.

Fuente: Elaboración propia en base a datos de PISAC (2015)

Además, el tiempo dedicado a tareas domésticas y de cuidado no remuneradas está negativamente asociado con el tiempo destinado a actividades para el mercado o de ocio (Munien y Ahmed, 2012; Rojas y Siles, 2014). La cantidad de horas que en promedio destinan las mujeres mayores de 15 años en áreas urbanas a tareas no remuneradas en el hogar disminuye cuando aumenta el ingreso per cápita del hogar, 
mientras que para los varones las horas medias destinadas a este tipo de actividad se mantienen relativamente constantes (Figura 1).

Gráfico 1. Horas promedio destinadas a trabajo doméstico y de cuidado no remunerado de personas mayores de 15 años, por grupo decílico de ingreso total del hogar per cápita y género

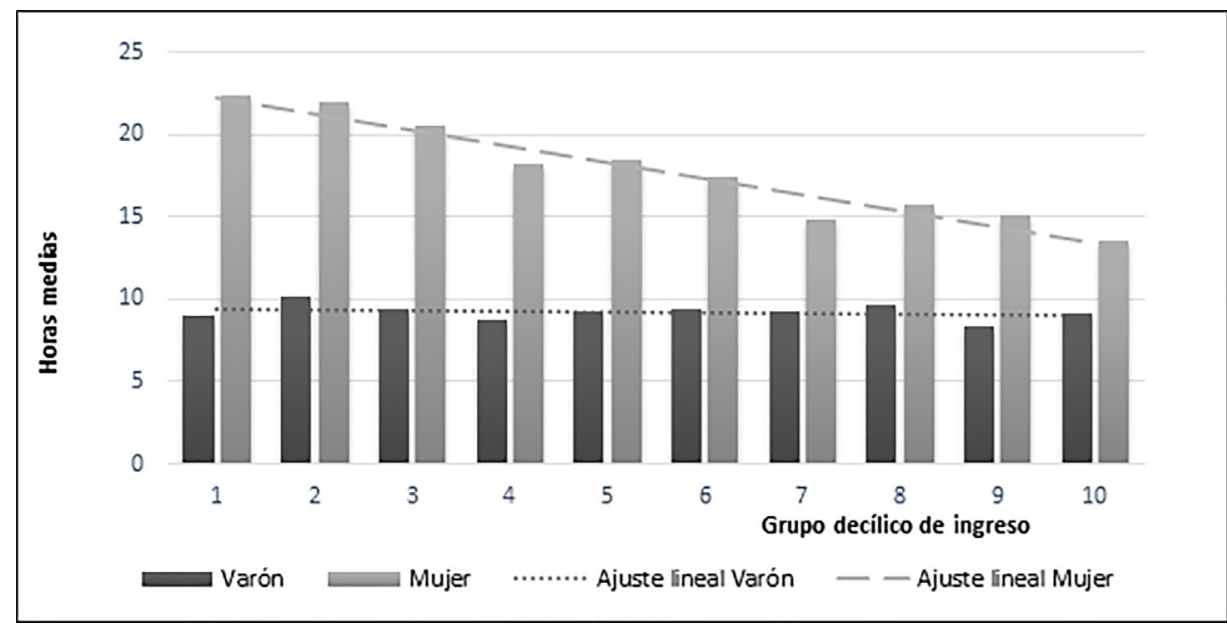

Fuente: Elaboración propia en base a datos de PISAC (2015)

\section{Una aproximación a la pobreza energética con enfoque de género}

La información estadística actualmente disponible en Argentina no ha sido diseñada para identificar y medir la pobreza energética y los diferentes aspectos que la integran. Sin embargo, el Censo, la EAHU y el ENES permiten analizar dos dimensiones claves para comenzar a entender la vulnerabilidad de los hogares frente a este fenómeno. Una dimensión es el acceso a servicios energéticos modernos y confiables, la cual puede ser analizada mediante la conexión y calidad del servicio y el tipo de tecnología utilizada. Otra dimensión es el acceso a bienes económicos que median en el consumo de energía para dar respuesta a satisfactores como la alimentación, el aseo personal, el confort térmico, la seguridad, el entretenimiento y comunicación. Adicionalmente, la ENES y EAHU relevan el uso del tiempo de los miembros del hogar y permiten comenzar a entender cómo la pobreza energética afecta de manera desigual a los integrantes del hogar según su género.

\subsection{La calidad del acceso a los servicios de energía}

Las bases de datos analizadas presentan información sobre conexión, calidad y tecnología de manera parcializada. En las bases del Censo y la EAHU, la conexión al servicio de gas puede medirse indirectamente a través de la variable "Combustible usado principalmente para cocinar". Las categorías de respuesta positiva incluyen el Gas de red y el Gas a granel, de tubo o garrafa, y las categorías de respuesta negativa se corresponden con el uso de Kerosene, leña o carbón. Las respuestas como Electricidad y Otros son consideradas como casos perdidos a los fines de estimar la tasa de conexión al servicio de gas (Tabla 2). La tecnología de este servicio, por otra parte, se encuentra definida por el tipo de servicio: gas por red o a 
granel, tubo o garrafa. A los fines de este estudio, se considera que el servicio de gas más confiable por su calidad y seguridad es el provisto a través de la red (Tabla 2). Por otro lado, la base de la ENES releva el acceso a los servicios energéticos preguntando directamente si la vivienda posee conexión a la red eléctrica y a la red de gas natural, distinguiendo si estas conexiones se encuentran en la vivienda, fuera de la vivienda en el terreno, o fuera del terreno. Además, la calidad del servicio eléctrico puede ser evaluada utilizando la variable "Presencia de problemas en el barrio con corte de luz por tiempo prolongado". Nuevamente, se considera que el acceso a servicios energéticos modernos y confiables ocurre cuando la conexión se encuentra en la vivienda y, en el caso del servicio eléctrico, cuando no hay problemas de corte de luz prolongados (3 o más horas) en el último año (Tabla 2).

Si bien la información que brinda el Censo es válida a nivel de departamento y su medición es sistemática, los datos recopilados a nivel urbano por la ENES brinda una noción más acabada que sobre el acceso a energía moderna y confiable al recopilar información sobre conexión y calidad de los servicios y, en un sentido parcial, sobre la tecnología utilizada para acceder a gas.

Tabla 2. Variables usadas para evaluar el acceso a servicios energéticos, según fuente de información.

\begin{tabular}{|l|c|c|c|}
\hline Dimensión & CENSO & EAHU & ENES \\
\hline Conexión & $\begin{array}{c}\text { Conexión a red } \\
\text { eléctrica en la } \\
\text { vivienda }\end{array}$ & NR & $\begin{array}{c}\text { Conexión a red } \\
\text { eléctrica en la } \\
\text { vivienda }\end{array}$ \\
\cline { 2 - 4 } & $\begin{array}{c}\text { Combustible usado } \\
\text { principalmente para } \\
\text { cocinar }\end{array}$ & $\begin{array}{c}\text { Combustible usado } \\
\text { para cocinar }\end{array}$ & $\begin{array}{c}\text { Conexión a red de } \\
\text { gas natural en la } \\
\text { vivienda }\end{array}$ \\
\hline Calidad & NR & NR & $\begin{array}{c}\text { Problemas en el } \\
\text { barrio con corte } \\
\text { de luz por tiempo } \\
\text { prolongado }\end{array}$ \\
\hline Tecnología & $\begin{array}{c}\text { Combustible usado } \\
\text { principalmente para } \\
\text { cocinar }\end{array}$ & Combustible usado para & cocinar \\
\hline
\end{tabular}

Nota: NR: información no recolectada.

Fuente: Elaboración propia a partir de INDEC $(2015,2014)$ y PISAC $(2015)$.

Según el Censo, en Argentina el 1,3\% de los hogares urbanos utiliza leña o carbón como principal combustible para cocinar. Este porcentaje se reduce a $0,4 \%$ en la EAHU. La base ENES no recopila esta información, pero el porcentaje de hogares con acceso a gas de red dentro de la vivienda $(65,3 \%)$ es similar al que presenta la EAHU $(64,7 \%)$ (Tabla 3).

El acceso a energía eléctrica según la base ENES alcanza al 98,1\% de los hogares, pero el $46 \%$ posee un servicio de baja calidad debido a cortes de luz prolongados. De esta forma, el porcentaje de hogares con acceso a energía eléctrica moderna y confiable se reduce a $53,1 \%$. 
Tabla 3. Porcentaje de hogares con acceso a servicios energéticos modernos y confiables en áreas urbanas, según fuente de información

\begin{tabular}{|l|c|c|c|}
\hline \multicolumn{1}{|c|}{ Dimensión } & CENSO & EAHU & ENES \\
\hline Acceso a servicio de energía eléctrica & 98,9 & NR & 53,1 \\
\hline Acceso a servicio de gas & 60,6 & 64,7 & 65,3 \\
\hline
\end{tabular}

Nota: NR: información no recolectada.

Fuente: Elaboración propia a partir de INDEC $(2015,2014)$ y PISAC $(2015)$.

\section{$5.2 \mathrm{El}$ acceso a bienes económicos que median en el uso de la energía}

En base al enfoque de las Necesidades Absolutas de la Energía se identificaron aquellos bienes económicos, recolectados por las bases de datos analizadas, que facilitan la satisfacción de necesidades básicas (Tabla 4). El uso de cada uno de estos bienes económicos puede estar asociado a la satisfacción de una o más necesidades, pero su tenencia no es una condición suficiente para la subsistencia de los hogares. Además, los bienes económicos identificados pueden no ser representativos de los usos y estrategias que aplican los hogares para satisfacer sus necesidades en los distintos entornos naturales y sociales del país. En este sentido, es necesario profundizar en las estrategias de supervivencia, usos y niveles de complementariedad y sustitución que poseen los bienes económicos para los hogares en distintas regiones del país.

La manera en que estas variables captan el acceso a los bienes económicos requiere algunas aclaraciones. En primer lugar, la variable "Procedencia del agua para beber y cocinar" permite identificar a los hogares que no tienen acceso a la red de agua potable ni cuentan con una perforación con bomba a motor. Este bien económico que utiliza energía eléctrica, facilita el acceso continuo a agua potable para satisfactores claves como la alimentación y el aseo personal.

En segundo lugar, las variables "Calefactores móviles/ por instalación fija" y "Aire acondicionado" fueron agrupadas, dado que su incidencia en la pobreza energética de los hogares depende del clima. Se supone que los hogares que acceden, al menos, a uno de estos equipos están cubriendo su principal necesidad térmica. En este sentido, el porcentaje de hogares con Calefactores en regiones cálidas como el NEA y el NOA, es de $44 \%$ y $51 \%$ respectivamente, mientras que, en regiones frías como Patagonia y Pampeana, este porcentaje alcanza al $96 \%$ y $91 \%$ de los hogares. Por último, tanto la base de datos del Censo como de la ENES consideran el teléfono móvil o celular como un bien del hogar, por lo que se supone que los hogares acceden a este bien si al menos uno de sus integrantes tiene un teléfono celular. 
Tabla 4. Variables utilizadas para identificar el acceso a bienes económicos que median en el uso de la energía, según fuente de información.

\begin{tabular}{|c|c|c|c|c|}
\hline Necesidades & Satisfactores & CENSO & EAHU & ENES \\
\hline \multirow{8}{*}{$\begin{array}{c}\text { Subsistencia, } \\
\text { protección, } \\
\text { libertad }\end{array}$} & \multirow{3}{*}{ Alimentación } & $\begin{array}{c}\text { Procedencia } \\
\text { del agua } \\
\text { para beber y } \\
\text { cocinar }\end{array}$ & $\begin{array}{l}\text { Procedencia } \\
\text { del agua }\end{array}$ & $\begin{array}{l}\text { Procedencia } \\
\text { del agua } \\
\text { para beber y } \\
\text { cocinar }\end{array}$ \\
\hline & & NR & NR & $\begin{array}{l}\text { Anafe o cocina } \\
\text { sin/ con horno }\end{array}$ \\
\hline & & Heladera & NR & $\begin{array}{l}\text { Heladera sin/ } \\
\text { con freezer }\end{array}$ \\
\hline & \multirow[t]{2}{*}{ Aseo personal } & $\begin{array}{l}\text { Procedencia } \\
\text { del agua } \\
\text { para beber y } \\
\text { cocinar }\end{array}$ & $\begin{array}{c}\text { Procedencia } \\
\text { del agua }\end{array}$ & $\begin{array}{l}\text { Procedencia } \\
\text { del agua } \\
\text { para beber y } \\
\text { cocinar }\end{array}$ \\
\hline & & NR & NR & $\begin{array}{c}\text { Termotanque/ } \\
\text { Calefón }\end{array}$ \\
\hline & \multirow{2}{*}{$\begin{array}{l}\text { Confort } \\
\text { térmico* }\end{array}$} & NR & NR & $\begin{array}{l}\text { Calefactores } \\
\text { móviles/ por } \\
\text { instalación fija }\end{array}$ \\
\hline & & NR & NR & $\begin{array}{c}\text { Aire } \\
\text { acondicionado }\end{array}$ \\
\hline & Seguridad & NR & NR & $\begin{array}{l}\text { Presencia de } \\
\text { servicio de } \\
\text { alumbrado } \\
\text { público en la } \\
\text { cuadra }\end{array}$ \\
\hline \multirow{4}{*}{$\begin{array}{c}\text { Entendimiento, } \\
\text { Participación, } \\
\text { Placer, Ocio, } \\
\text { Libertad }\end{array}$} & \multirow{4}{*}{$\begin{array}{l}\text { Entretenimiento } \\
\text { y comunicación }\end{array}$} & $\begin{array}{c}\text { Computadora } \\
\text { (de escritorio } \\
\text { o portátil) }\end{array}$ & NR & $\begin{array}{c}\text { Computadora } \\
\text { de escritorio/ } \\
\text { portátil }\end{array}$ \\
\hline & & $\begin{array}{c}\text { Teléfono } \\
\text { celular }\end{array}$ & NR & $\begin{array}{l}\text { Teléfono } \\
\text { celular }\end{array}$ \\
\hline & & NR & NR & $\begin{array}{l}\text { Servicio de } \\
\text { Internet }\end{array}$ \\
\hline & & NR & NR & $\begin{array}{c}\text { Televisión } \\
\text { común/ } \\
\text { Plasma/LCD }\end{array}$ \\
\hline
\end{tabular}

Nota: NR: información no recolectada.

Fuente: Adaptado de García-Ochoa (2014).

Como muestra la Tabla 5, el acceso de los hogares a bienes económicos que permiten hacer uso de la energía en zonas urbanas es menor a nivel país, según 
el Censo, que en los aglomerados relevados por la ENES. Por otra parte, los datos de la ENES muestran un bajo acceso de los hogares a bienes de entretenimiento y comunicación. Si bien estos datos en sí no reflejan una situación de pobreza energética, muestran que el acceso a energía en estos hogares no se traduce en el uso de tecnología que, en las sociedades modernas, puede condicionar la inclusión social de las personas.

Tabla 5. Porcentaje de hogares con acceso a los bienes económicos que median en el uso de la energía en áreas urbanas, según fuente de información.

\begin{tabular}{|c|c|c|c|c|}
\hline Satisfactores & Bienes económicos & CENSO & EAHU & ENES \\
\hline \multirow[t]{3}{*}{ Alimentación } & $\begin{array}{c}\text { Acceso a agua para beber } \\
\text { y cocinar por red de agua } \\
\text { o perforación con bomba a } \\
\text { motor }\end{array}$ & 98,0 & 99,4 & 98,1 \\
\hline & Anafe/ cocina sin/ con horno & NR & NR & 99,1 \\
\hline & Heladera & 95,3 & NR & 97,1 \\
\hline \multirow[t]{2}{*}{ Aseo personal } & $\begin{array}{c}\text { Acceso a agua para } \\
\text { beber y cocinar por red } \\
\text { de agua o perforación } \\
\text { con bomba a motor }\end{array}$ & 98,0 & 99,4 & 98,1 \\
\hline & Termotanque/ Calefón & NR & NR & 87,8 \\
\hline Confort térmico & $\begin{array}{c}\text { Calefactores móviles/ } \\
\text { por instalación fija o Aire } \\
\text { acondicionado }\end{array}$ & NR & NR & 83,8 \\
\hline \multirow{4}{*}{$\begin{array}{l}\text { Entretenimiento } \\
\text { y comunicación }\end{array}$} & Computadora & 49,3 & NR & 65,6 \\
\hline & Teléfono celular & 86,5 & NR & 88,3 \\
\hline & Servicio de Internet & NR & NR & 52,5 \\
\hline & Televisión & NR & NR & 97,2 \\
\hline Seguridad & $\begin{array}{c}\text { Presencia de servicio de } \\
\text { alumbrado público en la } \\
\text { cuadra }\end{array}$ & NR & NR & 95,7 \\
\hline
\end{tabular}

Nota: NR: información no recolectada.

Fuente: Elaboración propia a partir de INDEC $(2014,2015)$ y PISAC (2015).

\subsection{Una aproximación a la pobreza energética}

Como aproximación al problema de la pobreza energética en el país, el trabajo propone explorar el alcance y la intensidad del acceso de los hogares urbanos a servicios energéticos modernos y confiables y a bienes económicos básicos que consumen energía. Adicionalmente, se propone indagar sobre la relación entre este aspecto de la pobreza energética y el uso del tiempo en tareas domésticas y de cuidado no remuneradas. Para esto se utilizó la base de datos de la ENES, ya que permite un mayor acercamiento al problema, a través de diversas variables, que el resto de las bases analizadas.

Para evaluar el acceso de los hogares urbanos se agruparon las variables 
identificadas en los apartados previos -conexión y calidad de los servicios (Tabla 3 ) y bienes económicos (Tabla 5)- en un índice. Este índice se construyó mediante la suma simple de las variables, las que adoptan el valor 1 si el hogar accede al atributo o bien económico en cuestión y valor 0 en caso contrario, por lo que a mayor valor del índice, menor pobreza energética. En esta fórmula, cada variable recibe igual ponderación, dado que no se cuenta con información complementaria que permita evaluar su pertinencia individual en la pobreza energética. No obstante, en el agregado, el índice otorga mayor peso a la tenencia de bienes económicos por sobre el acceso a servicios, ya que la primera engloba once variables y el acceso a servicios solo dos. Si bien las propuestas metodológicas de indicadores para medir la pobreza energética a nivel internacional son múltiples y diversas (Calvo et al., 2018), García-Ochoa y Graizbord (2016) y Nussbaumer, Bazilian, Modi y Yumkella (2012) proponen índices multidimensionales a partir de variables que reflejan el acceso a servicios energéticos y la posesión de bienes económicos y utilizan funciones de agregación que otorgan distintos pesos relativos a cada una de las variables.

Este índice muestra que sólo el $20 \%$ de los hogares urbanos del país relevados en la ENES cuenta con acceso a energía moderna y confiable y a bienes económicos básicos, y el $61,7 \%$ de los hogares accede a entre 10 y 12 atributos o bienes. En un país donde poco más del $60 \%$ de los hogares urbanos accede a gas de red y casi la mitad a un servicio eléctrico de calidad -sin cortes de luz-, no es extraño que una gran proporción de hogares se encuentre expuesta a estas dimensiones de la pobreza energética ${ }^{5}$.

Al analizar la relación entre este índice y las horas medias semanales destinadas al trabajo doméstico y de cuidados no remunerados según género (Figura 2) se observa, por un lado, que las mujeres destinan un tiempo significativamente mayor que los varones a este tipo de trabajo. Por otro lado, el gráfico muestra que las horas destinadas a estas tareas disminuyen cuando el índice de acceso a servicios energéticos y bienes económicos aumenta. En otras palabras, cuanto mayor es el acceso (o menor la pobreza energética en los aspectos analizados), menor es el tiempo destinado por mujeres y varones al trabajo doméstico y de cuidado no remunerado ${ }^{6}$. Esta relación negativa entre las variables y su incidencia desigual según el género se mantiene aun cuando se controla estadísticamente el impacto del ingreso total per cápita.

5 Para evaluar la consistencia interna del índice de Pobreza Energética se aplicó el método Alfa de Cronbach, el cual refleja una consistencia pobre cuando su valor está por debajo de 0,5 y aceptable cuando el valor es mayor a 0,7. El análisis de las variables que integran el índice de Pobreza Energética adoptó un valor de 0,632, lo que plantea el desafío de seguir robusteciendo la construcción y el relevamiento de los indicadores que integran el índice.

6 El coeficiente de correlación (lineal) de Pearson entre estas variables es estadísticamente significativo a un nivel de significancia de 0,01 . Este coeficiente es negativo tanto para la población de mujeres como para la de varones, pero mientras para las mujeres la intensidad de esta relación es $-0,16$, para los varones es $-0,04$. 
Gráfico 2. Horas semanales promedio destinadas a actividades de trabajo doméstico y de cuidados no remunerado, según género y nivel de pobreza energética. Personas mayores de 15 años.

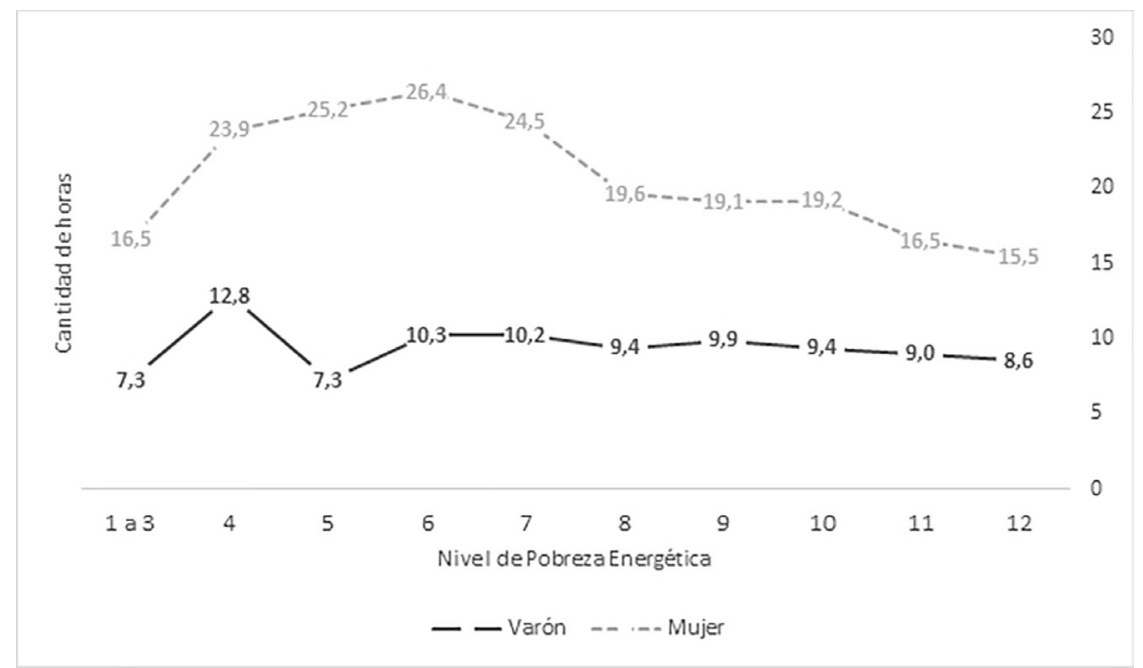

Fuente: Elaboración propia a partir de PISAC (2015).

Los factores que inciden en el acceso a servicios energéticos y bienes económicos toman visibilidad cuando este indicador se desagrega por tipo de satisfactor asociado al uso de la energía (Tabla 6). La calidad de acceso al servicio de energía eléctrica y la falta de acceso a bienes económicos vinculados a entretenimiento y comunicación afecta a una gran proporción de hogares. En un menor porcentaje, pero con mayor incidencia en condiciones básicas de bienestar, se destaca las privaciones asociadas al aseo personal. Esta situación cuestiona el consumo total de energía que realizan estos hogares como reflejo de sus necesidades energéticas reales. A su vez, resulta importante profundizar el análisis de los satisfactores en relación con el género y de qué forma la falta de uno de ellos acrecienta las desigualdades en el uso del tiempo destinado a trabajo doméstico y de cuidado.

Tabla 6. Porcentaje de hogares que presentan al menos una privación, según satisfactor.

\begin{tabular}{|l|c|}
\hline \multicolumn{1}{|c|}{ Satisfactores } & $\%$ \\
\hline Acceso a energía & 62,5 \\
\hline Alimentación & 5,2 \\
\hline Aseo personal & 13,5 \\
\hline Confort térmico & 15,9 \\
\hline Entretenimiento y comunicación & 52,3 \\
\hline Seguridad & 4,2 \\
\hline
\end{tabular}

Fuente: Elaboración propia a partir de PISAC (2015). 


\section{Algunas conclusiones}

La pobreza energética se ha consolidado a nivel internacional como un concepto relevante para comprender un fenómeno complejo que a través de distintos canales afecta a hogares en distintos países y condiciones socio-económicas. Se trata, sin embargo, de un término con vacíos conceptuales y metodológicos, escasamente articulado con los conceptos de pobreza, estrategias de supervivencia, vulnerabilidad y exclusión social. Además, la bibliografía existente aún no explica de manera acabada cómo interactúan los distintos factores que condicionan la pobreza energética y cómo este fenómeno incide en la realización de trabajos remunerados y no remunerados, y en el bienestar de los miembros del hogar. En este sentido, es importante explorar las consecuencias que se derivan de la pobreza energética y de cada una de sus dimensiones desde un enfoque de género, de forma tal de visibilizar desigualdades y formular políticas públicas que atiendan tanto las causas como las consecuencias del problema.

Las bases de datos disponibles en Argentina no ofrecen información adecuada para medir de manera confiable el alcance y la intensidad de la pobreza energética a nivel nacional e identificar con claridad los factores que la determinan. Sin embargo, a nivel urbano, es posible corroborar que la conexión a los servicios energéticos no es un factor central de la pobreza energética en el país. Por el contrario, la baja calidad del servicio y el tipo de tecnología utilizada para acceder al servicio de gas condicionan significativamente el acceso a energía moderna y confiable. Sumada la falta de acceso a bienes económicos básicos que median en el consumo de energía, los datos sugieren la presencia de este fenómeno, con mayor o menor intensidad, en la mayoría de los hogares del país.

La falta de acceso a bienes económicos como computadora e internet $-34,4 \%$ y $47,5 \%$ respectivamente- posee un peso importante en el alcance de este fenómeno; sin embargo, los teléfonos móviles "inteligentes" podrían estar supliendo la ausencia de estos bienes, aunque se desconoce el nivel de acceso de los hogares a este tipo de teléfonos. Además, el papel de estos bienes en la satisfacción de necesidades está marcado por la modernidad, por lo que su incidencia real en el bienestar de las personas debería ser evaluada considerando su entorno geográfico y cultural. Por el contrario, la falta de acceso a bienes económicos destinados al aseo personal $(13,5 \%)$ y el confort térmico $(15,9 \%)$ inciden claramente en el bienestar de las personas. Esto muestra la importancia de conocer las estrategias energéticas de los hogares, y los usos y niveles de complementariedad y sustitución que tienen los bienes económicos en distintas regiones del país, para identificar aquellos con mayor incidencia en la pobreza energética.

Por otro lado, falta información en relación a los usos del tiempo y su desagregación en tareas domésticas y de cuidado no remuneradas que hacen uso de la energía, para medir los tiempos que se destinan a diferentes tareas como lavar, planchar, cocinar, etc. Dicha información, aportaría en el entendimiento de las estrategias que aplican los hogares en sus entornos, en relación a los hábitos, al acceso a bienes económicos y de qué forma afecta al género. Esto permitiría pensar políticas energéticas que tengan una transversalización de género atendiendo a las 
particularidades de los hogares usuarios.

\section{Referencias bibliográficas}

Belaïd, F. (2018). Exposure and risk to fuel poverty in France: Examining the extent of the fuel precariousness and its salient determinants. Energy Policy, 114, 189-200. doi: 10.1016/j.enpol.2017.12.005

Boardman, B. (1991). Fuel poverty: from cold homes to affordable warmth. Londres, Inglaterra: Belhaven Press.

Boltvinik Kalinka, J. (2005). Ampliar la mirada. Un nuevo enfoque de la pobreza y el florecimiento humano (Tesis doctoral). Centro de Investigaciones y Estudios Superiores en Antropología Social-Occidente, Jalisco, México. Recuperado de www.julioboltvinik.org/documento/Tesis\%20Ampliar\%20 la\%20mirada\%20PDF/Vol\%20I/Cap\%C3\%ADtulo\%205.\%20Max\%20Neef. pdf.

ECOWAS (2015). Policy for Gender Mainstreaming in Energy Access, Economic Community of West African States. Recuperado de: ecowgen.ecreee.org/ wp-content/uploads/2015/11/ECOWAS-Policy-for-Gender-Mainstreamingin-Energy-Access2.pdf

García-Ochoa, R. (2014). Pobreza energética en América Latina. Santiago de Chile, Chile: CEPAL. Recuperado de repositorio.cepal.org/bitstream/ handle/11362/36661/S2014039_es.pdf;jsessionid=712F64DD13A43C204C AADC4A57D743BC?sequence=1.

García-Ochoa, R. y Graizbord, B. (2016). Caracterización espacial de la pobreza energética en México. Un análisis a escala subnacional. Economía, sociedad y territorio, 16(51), 289-337.

González Pijuan, I. (2017). Desigualdad de género y pobreza energética. Un factor de riesgo olvidado. Cataluña, España: Ingeniería sin Fronteras. Recuperado de esf-cat.org/wp-content/uploads/2017/09/ESFeres17-PobrezaEnergeticai DesigualdadGenero.pdf

Healy, J. y Clinch, J. P. (2002). Fuel Poverty in Europe: A Cross-Country Analysis Using a New Composite Measurement. Environmental Studies Research Series Working Papers.

INDEC [Instituto Nacional de Estadísticas y Censos]. (2014). Encuesta Anual de Hogares Urbanos, $3^{\circ}$ Trimestre del año 2013. Recuperado de www.indec. gob.ar/bases-de-datos.asp?solapa=7

INDEC [Instituto Nacional de Estadísticas y Censos]. (2015). Censo Nacional de Población, Hogares y Viviendas, año 2010. Recuperado de redatam.indec. gob.ar.

Khamati-Njenga, B. y Clancy, J. (2003). Concepts and Issues in Gender and Energy, Energia, 1-77. 
Lewis, P. (1982). Fuel poverty can be stopped. Bradford, Inglaterra: National Right to Fuel Campaign.

Liddell, C. y Morris, C. (2010). Fuel poverty and human health: a review of recent evidence, Energy Policy, 38, 2987-2997. doi: 10.1016/j.enpol.2010.01.037

Maceira, V. (2015). Un abordaje teórico-metodológico para la investigación de la estructura, la movilidad social y las condiciones de vida: la propuesta ENESPISAC. Revista Latinoamericana de Metodología de las Ciencias Sociales, $5(2)$.

Max Neef, M., Elizalde A. y Hopenhayn M. (1986). Desarrollo a escala humana Opciones para el futuro. Recuperado de: habitat.aq.upm.es/deh/.

Mininni G. (2015). Gender and energy issues in the global south: implications for the post-Millennium Development Goals agenda after 2015. Recuperado de www.lancaster.ac.uk/luminary/issue5/issue5article4.htm

Moore, R. (2012). Definitions of fuel poverty: Implications for policy. Energy Policy, 49, 19-26. doi: 10.1016/j.enpol.2012.01.057

Modi, V., Mc Dade, S., Lallement, D. y Saghir, J. (2005). Energy Services for the Millennium Development Goals. Recuperado de lutw.org/wp-content/ uploads/Energy-services-for-the-millennium-development-goals.pdf

Munien S. y Ahmed F. (2012). A gendered perspective on energy poverty and livelihoods - Advancing the Millennium Development Goals in developing countries. Agenda, 26, 112-123. doi: 10.1080/10130950.2012.674252.

Nussbaumer, P., Bazilian, M., Modi, V. y Yumkella, K.K. (2012). Measuring Energy Poverty: Focusing on What Matters. Renewable and Sustainable Energy Reviews, 16(1), 231-243.

Papada, L. y Kaliampakos D. (2016). Measuring energy poverty in Greece. Energy Policy, 94, 157-165. doi: 10.1016/j.enpol.2016.04.004.

Programa de Investigación sobre la Sociedad Argentina Contemporánea [PISAC]. (2015). Encuesta Nacional sobre la Estructura Social, 2014/2015. Recuperado de pisac.mincyt.gob.ar/datos.php.

Richardson, P. (1978). Fuel Poverty: A Study of Fuel Expenditure among Low Income Council Tenants. York, Canadá: University of York.

Rojas, A. V. y Siles, J. (2014). Guía sobre Género y Energía para Capacitadoras(es) y Gestoras(es) de Políticas Publicas y Proyectos. ENERGIA, OLADE, IUCN. Recuperado de portals.iucn.org/library/sites/library/files/ documents/2014-061.pdf

Scott, J. (1993). El género: una categoría útil para el análisis histórico. En M. C. Cangiano y L. Du Bois. (Eds.). De mujer a género. Teoría, interpretación y práctica feminista en las ciencias sociales. Buenos Aires, Argentina: Centro 
Editor de América Latina.

Sen, A. (1981). Poverty and Famines. An Essay on Entitlement and Deprivation. Oxford, Inglaterra: Clarendon Press.

Simcock, N. y Mullen, C. (2016). Energy demand for everyday mobility and domestic life: Exploring the justice implications. Energy Research and Social Science, 18, 1-6. doi: 10.1016/j.erss.2016.05.019.

Skutsch, M. y Wamukonya, N. (2001). Gender angle to the climate change negotiations. Energy \& Environment, 13(1), 115-124. doi: 10.1260/0958305021501119

Sovacool, B. K., Sidortsov, R. V., y Jones, B. R. (2014). Energy Security, Equality and Justice. Londres, Inglaterra: Routledge.

Thomson, H., Snell, C. y Liddell, C. (2016). Fuel poverty in the European Union: a concept in need of definition? People Place and Policy, 10(1), 5-24. doi: 10.3351/ppp.0010.0001.0002.

Thomson, H., Snell, C. y Bouzarovski, S. (2017). Health, well-being and energy poverty in Europe: A comparative study of 32 European countries. International Journal of Environmental Research and Public Health, 14, 1-17. doi: 10.3390/ijerph14060584.

Townsend, P. (2006). Introduction. En Rio Group. Expert Group on Poverty Statistics. Compendium of best practices in poverty measurement. Río de Janeiro, Brasil: CEPAL. Recuperado de www.cepal.org/publicaciones/xml/3/26593/ rio_group_compendium.pdf

United Nations. (2018). Accelerating SDG7. Achievement Policy Briefs in Support of the First SDG7. Review at the UN High-Level Political Forum 2018. Recuperado de sustainabledevelopment.un.org/content/documents/18041SDG7_Policy_ Brief.pdf 